Balance de las políticas de fomento para la producción de contenidos de ficción en alta definición en la Argentina (2011-2015)

Ornela Carboni

Tram[p]as de la comunicación y la cultura (N. $\left.{ }^{\circ} 84\right)$, e037, 2019

ISSN 2314-274X | https://doi.org/10.24215/2314274xe037

http://perio.unlp.edu.ar/ojs/index.php/trampas

FPyCS | Universidad Nacional de La Plata

La Plata | Buenos Aires | Argentina

\title{
BALANCE DE LAS POLÍTICAS DE FOMENTO PARA LA PRODUCCIÓN DE CONTENIDOS DE FICCIÓN EN ALTA DEFINICIÓN EN LA ARGENTINA (2011-2015)
}

BALANCE OF PROMOTION POLICIES FOR THE PRODUCTION

OF FICTION CONTENTS IN HIGH DEFINITION IN ARGENTINA (2011-2015)

\author{
Ornela Carboni \\ ornelacarboni@yahoo.com.ar \\ https://orcid.org/0000-0002-3968-6455 \\ Universidad Nacional de Quilmes \\ Argentina
}

\begin{abstract}
Resumen
Abstract

Desde el enfoque de la economía política

From the perspective of the political de la comunicación, este estudio analiza, economy of communication, this study en el período 2011-2015, las políticas de analyzes, during 2011-2015, the policies fomento a la producción de contenidos de ficción, en el marco de la aplicación de la Ley 26.522 de Servicios de Comunicación Audiovisual y de la implementación de la Televisión Digital Abierta (TDA). to promote the production of fiction content, from the application of the Ley 26.522 de Servicios de Comunicación Audiovisual and the implementation of Open Digital Television.
\end{abstract}

Palabras clave | políticas de comunicación, televisión digital abierta, producción de contenidos, Estado

Keywords | communication policies, digital television, content production, State 


\title{
BALANCE DE LAS POLÍTICAS DE FOMENTO
}

\section{PARA LA PRODUCCIÓN DE CONTENIDOS \\ DE FICCIÓN EN ALTA DEFINICIÓN}

EN LA ARGENTINA (2011-2015)

\author{
Por Ornela Carboni
}

\section{Introducción}

El presente estudio analiza, desde el enfoque de la economía política de la comunicación, las medidas de fomento para la producción de contenidos de ficción en alta definición destinados a la televisión argentina durante el período 2010-2015. Los incentivos a la producción audiovisual se enmarcaron en la Ley Nacional 26.522 de Servicios de Comunicación Audiovisual (LSCA) y en la implementación de la televisión digital terrestre (TDT) durante el gobierno de Cristina Fernández de Kirchner (2007-2011, 2011-2015).

En la Argentina, las politicas regulatorias para los medios de comunicación vivenciaron durante 2009 una etapa inédita. El inicio de la transformación fue la promulgación, en octubre de 2009, de la LSCA, que reemplazó al Decreto Ley 22.285 de Radiodifusión, de 1980. La normativa fue considerada de avanzada debido a que estableció límites a la concentración, promovió el desarrollo de los sectores sin fines de lucro, estimuló la producción nacional de contenidos para la radio y televisión, y reconfiguró la autoridad de aplicación. Sin embargo, el gobierno que concretó su sanción provocó dilaciones en su implementación, no aplicó todos los artículos de la regulación y, en algunos casos, lo hizo de modo sesgado y dispar de acuerdo a intereses alejados de los principios rectores de la normativa. 
En 2015, a partir de la llegada al gobierno del espacio Cambiemos, liderado por Mauricio Macri, la dirección de las políticas de comunicación modificó su curso y sufrió un cimbronazo que obturó los avances de la ley en relación con el derecho a la comunicación. En sus primeros días de gobierno, Macri firmó un Decreto de Necesidad y Urgencia (DNU) que supeditó a la Autoridad Federal de Servicios de Comunicación Audiovisual (AFSCA) y a la Autoridad Federal de Tecnologías de la Información y la Comunicación (AFTIC) a la órbita del Ministerio de Comunicaciones. Posteriormente, mediante el Decreto 267 $(2015)^{1}$ se desintegraron la AFSCA y la AFTIC, y se creó en su lugar el Ente Nacional de Comunicaciones (ENaCom), un organismo que regula el sector de las telecomunicaciones y los servicios de comunicación audiovisual con el supuesto objetivo de impulsar la convergencia tecnológica. Asimismo, en julio de 2017, a través del Decreto 513, el Ministerio de Comunicaciones fue desintegrado y la cartera pasó a manos del Ministerio de Defensa.

Este cambio de dirección revivió el debate sobre las tensiones entre la libertad de expresión versus la libertad de empresa y entre la concentración del mercado versus la garantía a la diversidad y al pluralismo informativo en el escenario audiovisual. Tales aspectos no serán objeto de nuestro análisis pero resulta válido no soslayar que estas tensiones cobran mayor o menor dinamismo dependiendo de la coyuntura.

El plan de fomento a la producción de contenidos audiovisuales acompañó el desarrollo del Sistema Argentino de Televisión Digital Terrestre (SATVD-T) impulsado por el Ministerio de Planificación Federal (MinPlan). En 2009, el Estado argentino había seleccionado la norma japonesa para la TDT, en un gesto político con Brasil. Los objetivos de las politicas para la TDT apuntaron a desarrollar la infraestructura de la red, garantizar el acceso material, generar empleo y estimular la producción de contenidos nacionales.

En este trabajo se incursiona en la planificación y en el estímulo a la producción de contenidos audiovisuales en alta definición durante el periodo 2010-2015 y se focaliza en la convocatoria para las series de ficción en alta definición (Full HD), que tenía entre sus objetivos contribuir a la federalización de la producción de contenidos televisivos y revertir la centralización en la Ciudad de Buenos Aires. 
El estudio, de carácter exploratorio y descriptivo, se concentra en abordar las medidas impulsadas para fomentar la producción de contenidos audiovisuales de ficción y en evaluar el impacto de las regulaciones para transformar la lógica centralista que predomina en la producción-emisión televisiva nacional y las posibilidades de subsistencia de producción local sin apoyo estatal. En esta dirección, se estudian, específicamente, las regulaciones y los concursos destinados a financiar los productos de ficción en alta definición para la televisión abierta nacional, se aborda la articulación entre las distintas fases de la cadena de valor y se evalúan los objetivos de inclusión y de federalismo.

Metodológicamente, se efectuó la revisión de las resoluciones que convocaban a los concursos y sus principales cláusulas, y se elaboró una grilla que detalla las ficciones producidas y emitidas bajo esta modalidad, y las productoras beneficiarias. Se destaca, al respecto, que los concursos se iniciaron en 2011 y concluyeron en 2014; mientras que en 2015 solo se realizaron tres telefilms.

La exposición seguida en el trabajo se organiza en cuatro apartados. En primer lugar, se explica la perspectiva teórica desde la cual se aborda el estudio y se profundiza en el concepto de industria televisiva. A continuación, se expone el contexto histórico de inserción del objeto de estudio para comprender sus dinámicas. Luego, se focaliza sobre la producción de los contenidos de ficción de alta calidad y, por último, se explica la fase de transmisión en la plataforma de la TDA, diseñada por el Estado Nacional en el período analizado. Como cierre, se sistematizan los principales hallazgos de la investigación.

\section{La televisión como industria cultural}

La perspectiva teórica de la investigación se centra en la economía politica de la comunicación, que estudia las actividades industrializadas de la cultura y las relaciones sociales, con énfasis en las relaciones de poder, que mutuamente constituyen la producción, la distribución y el consumo de los recursos de comunicación (Mosco, 2006). Martín Becerra (2011) sostiene que dichas actividades están constituidas históricamente pero que son, a la vez, constituyentes, puesto que son estructuradas por la historia politica, cultural y económica de cada país al tiempo que son estructurantes de cada sociedad. 
En esta dirección, el presente estudio retoma las relaciones que se establecen entre el gobierno, las productoras de contenidos y los canales de televisión, los procesos de concentración / desconcentración de los mercados mediáticos, las políticas regulatorias y el fomento a la producción de contenidos, al tener en cuenta la relación entre la producción material y simbólica de los bienes culturales.

Las industrias culturales producen y distribuyen mercancias con contenidos simbólicos, destinadas a los mercados de consumo, con una función de reproducción ideológica y social (Zallo, 1988). Tales industrias producen mercancías que tienen un doble valor: económico -reproducción del capitaly simbólico -transmisión de valores e identificaciones sociales- (Pasquali, 1991).

La reestructuración de la industria televisiva, los presagios sobre su posible fin o sus readecuaciones a las formas actuales de hacer y de ver televisión, ameritan entender sus lógicas de producción y de funcionamiento como, también, el interés de los gobiernos por estimular la producción nacional de contenidos para resguardar los valores y las costumbres del país.

\section{La televisión y sus marcas indelebles}

La televisión en la Argentina se consolidó en la década del sesenta bajo un sistema privado, comercial y competitivo (Becerra \& Mastrini, 2009) en el que la televisión estatal desempeñó un papel marginal. Esta fisonomía no perdió vigencia. En la actualidad, es un mercado mediano y concentrado bajo el duopolio conformado por El Trece -Grupo Clarín, con posición dominante en TV paga- y por TELEFE, que en 2016 el Grupo VIACOM compró al Grupo Telefónica. Este último presenta una posición dominante en el sector de las telecomunicaciones en la Argentina. La TV paga alcanza una penetración del $80 \%$ e los hogares mientras que la TDA presenta una cobertura sobre el $85 \%$ de la población con una adopción en 12\% de los hogares conectados (Carrier y asociados, 2016).

Con el objetivo de ampliar la oferta de señales en abierto, en 2010 el gobierno desarrolló una plataforma audiovisual a través de cuatro canales (22, 23, 24 y 25) en Ultra High Frequency (UHF) que fueron otorgados a Radio y Televisión 
Argentina (RTA) Sociedad del Estado. Dicha plataforma, declarada de interés público, alberga un conjunto de señales (públicas y privadas) que tienen un permiso precario para operar. Estas autorizaciones se otorgaron de manera discrecional y están justificadas ante la ausencia del plan técnico que le correspondía realizar a la AFSCA.

De acuerdo al sitio oficial de la TDA, en 2015, la plataforma contaba con un paquete de 16 señales de carácter nacional: siete eran públicas (TV Pública -Canal 7-, Encuentro, Paka Paka, Ta Te Ti, Incaa TV, TecTV, DeporTV), seis eran privadas (Rock and Pop, Arpeggio, ViajarTV, $360^{\circ}, \mathrm{C} 5 \mathrm{~N}$ y CN23), una era del tercer sector (Construir) y dos eran internacionales (Telesur y RT). Además, diez de ellas se crearon para la plataforma de la TDT. Luego, algunas se incluyeron en la oferta de señales de los sistemas de televisión paga.

En cuanto al área de cobertura, en el caso de la Ciudad Autónoma de Buenos Aires (CABA) y el Gran Buenos Aires se consolidaron 13 señales, de las cuales nueve eran de carácter privado y cuatro públicas. En el sector privado, se destacan las principales señales de la televisión abierta (TELEFE Canal 11, Canal 9, América 2 Canal 2, El Trece Canal 13) a las que sumaron Telemax, C5N, One TV, Canal 26 y A24. Las emisoras públicas, en tanto, son ACUA Mayor, ACUA federal, Digo TV y TV Universidad.

En 2013, la AFSCA autorizó a 33 televisoras de baja potencia a emitir en analógico con un permiso precario pero no las habilitó para subir la señal a la plataforma. Solo se permitió la transmisión de la señal Construir TV del Sindicato de la Construcción, con alcance nacional, y de las señales universitarias con alcance local. Además, en diciembre de 2015, se aprobó la licencia para los canales comunitarios de baja potencia Barricada TV, Urbana TeVé y Pares TV.

La generación de contenidos atractivos se consideró central para motorizar el pasaje hacia el universo digital y para romper con la concentración de la producción en el centro de Buenos Aires. El artículo 65 de la LSCA indicaba que los servicios de radiodifusión abierta debian emitir un mínimo de $60 \%$ de producción nacional y un mínimo de 30\% de producción propia. Para cumplir con tal directriz se establecieron programas liderados por el Instituto Nacional de Cine y Artes Audiovisuales (INCAA), el MinPlan, la Universidad Nacional de San Martín (UNSAM) y el Consejo Interuniversitario Nacional (CIN). 
Las producciones ganadoras de los concursos se integraron en el Banco de Contenidos Universales Argentino (BACUA), una red digitalizada para preservar la memoria audiovisual de la nación y para abastecer de contenidos de alcance universal que pueden ser utilizados por canales públicos, privados o del tercer sector. Asimismo, se podian visualizar vía streaming mediante la plataforma Odeon creada en noviembre de 2015, actualmente http://www.cine.ar. La misma es gestionada por el INCAA y cuenta con 900 horas de contenidos a los que se accede solamente desde la Argentina.

\section{Fomento a la producción de contenidos digitales 2011-2015}

Desde la sanción de la LSCA y la implementación de la Televisión Digital Abierta (TDA), uno de los vértices de las políticas de comunicación se focalizó en el fomento a la producción nacional de contenidos audiovisuales. Esta medida se orientó a una serie de objetivos. En primer lugar, buscó generar un reservorio para dotar de contenidos a las nuevas señales digitales cuyo surgimiento debía efectivizarse con la implementación de la TDA. Para almacenar y para administrar los productos audiovisuales se creó el BACUA. En segundo lugar, se proponía revertir el centralismo de Buenos Aires en la producción y en la emisión de contenidos que se concentra en dos emisoras TELEFE (Canal 11) y Artear (Canal 13), señales que en 2011 mantenían aproximadamente el 70\% de los contenidos retransmitidos en el país (AFSCA, 2012). Estos porcentajes no se alteraron sustancialmente en los años estudiados. Además, de los canales de televisión, en CABA se ubican las principales productoras de ficciones: Pol-ka, Central Park, Dori Media, RGB, ON TV, Underground, Endemol Argentina, Cris Morena Group, LC Acción Producciones, Ideas del Sur.

Las iniciativas intentaban fomentar la producción nacional de contenidos audiovisuales, atenuar el histórico centralismo de Buenos Aires y establecer una televisión federal e inclusiva mediante la creación de capacidades productivas en todo el país. Para ello, en 2010 se aprobó el Plan Operativo de Fomento y Promoción de Contenidos Audiovisuales Digitales. Desde entonces, y hasta 2014 inclusive, el MinPlan, la UNSAM, el CIN y el INCAA han diseñado e impulsado diversos concursos nacionales para fomentar la producción. 
Los concursos estuvieron destinados a actores con intereses en el sector audiovisual. La segmentación de las convocatorias se realizó en función de los siguientes aspectos: formatos (documentales, ficciones, entretenimiento), antecedentes en producción de los postulantes, presupuesto y futuras formas de distribución.

En esta dirección, se desarrollaron concursos nacionales (series de documentales para señales públicas, series de documentales y ficciones para productoras con antecedentes, series de animaciones nacionales, series de ficción para señales públicas), concursos federales (series de ficciones, de ficciones temáticas y de documentales federales, unitarios federales "Nosotros", series de animaciones y de animaciones temáticas federales, programas de piso para señales públicas) y concursos de series de ficción en alta definición (Full HD) los cuales son objeto del presente análisis.

En 2015 no hubo concursos, a excepción de tres telefilms temáticos.

\section{La fase de producción de las series en Full HD}

La convocatoria para las series de ficción en Full HD estuvo dirigida a los canales de televisión abierta. Las señales podían presentarse solas o en asociación con productoras con antecedentes, esto es, productoras con al menos una producción audiovisual de duración superior a los treinta minutos y exhibida en salas cinematográficas o en canales de televisión abierta o de cable. Entre 2010-2015 se realizaron cuatro ediciones y las producciones contaron con los presupuestos más elevados financiados con dinero público.

Los proyectos fueron encabezados por productoras (televisivas o cinematográficas) que se asociaron con una de las emisoras televisivas de CABA -con excepción de seis ficciones-, en una dinámica que nos introduce en la dicotomía entre una televisión inclusiva y federal versus una televisión centralista y porteña.

Los proyectos seleccionados debían ser exhibidos por los canales de televisión abierta en el prime time televisivo, establecido de lunes a viernes, de 20 a 24 horas. Todos los contenidos se incorporarian al BACUA y se estipulaba que los productores / directores debían ceder los derechos de propiedad intelectual al Estado nacional, que retenía los derechos patrimoniales o de explotación en todos los soportes. Sin embargo, los canales de televisión abierta y/o las 
productoras ganadoras conservaban para sí la comercialización internacional de la obra por 24 meses, excepto en Iberoamérica. Por último, se establecía que si la presentación era realizada por una productora asociada a un canal de televisión abierta debía anexarse el instrumento legal que indicara la relación entre las partes.

Durante 2011-2015, se financiaron 50 series de ficción en HD, que oscilaron entre los ocho y los trece capítulos. Algunas han sido estrenadas en canales de televisión abierta, seis (6) fueron emitidas por canales que no conforman la región CABA y AMBA, y dos (2) se ofrecieron en línea en la página de Contenidos Digitales Abiertos (CDA). Los datos relevados indican que del total de producciones financiadas, ocho (8) presentan anomalias: sobre seis no hay datos disponibles, una (Todos comen, a cargo de la productora Atuel) solo ofrece la visualización de un trailer y otra (Estocolmo) eludió la proyección en la televisión abierta argentina y se estrenó en Netflix (Ver Anexo).

En 2011 se financiaron diez (10) series. Cada una de las ficciones ganadoras recibió un presupuesto total de $\$ 4.550 .000$ (cuatro millones quinientos cincuenta mil), a razón de $\$ 350.000$ (trescientos cincuenta mil) por cada uno de los trece capítulos, de 48 minutos de duración. En 2012 y 2013 se desarrolló la segunda y tercera edición de proyectos de series de ficción, con los mismos objetivos que la primera pero con un incremento en el presupuesto asignado a cada una de ellas y en la cantidad de propuesta seleccionadas, 12 y 16 respectivamente. En 2014 se convocó la cuarta edición. La dinámica del concurso se modificó porque se desdobló en cuatro categorias (histórica, social, comedia y costumbrista) y se financiaron tres series por cada una de las categorias, lo que arroja un total de doce ficciones.

El siguiente gráfico muestra el porcentaje de ficciones emitidas por cada emisora entre 2011-2015. De las 50 ficciones financiadas, siete no han sido estrenadas o no se registran datos de su ejecución. De las 43 restantes, $28(65 \%)$ fueron emitidas por señales privadas y $12(28 \%)$ por emisoras públicas, dos (4\%) mediante la plataforma cda.gob.ar (que en diciembre de 2016 dejó de estar operativa) y una lo hizo solo por la plataforma privada transnacional Netflix. Además, del total de ficciones efectivamente emitidas casi $82,5 \%$ se estrenó en la región CABA y AMBA, y $17,5 \%$ en señales provinciales, lo que muestra el fuerte centralismo tanto en la producción como en la emisión de los contenidos audiovisuales televisivos. 


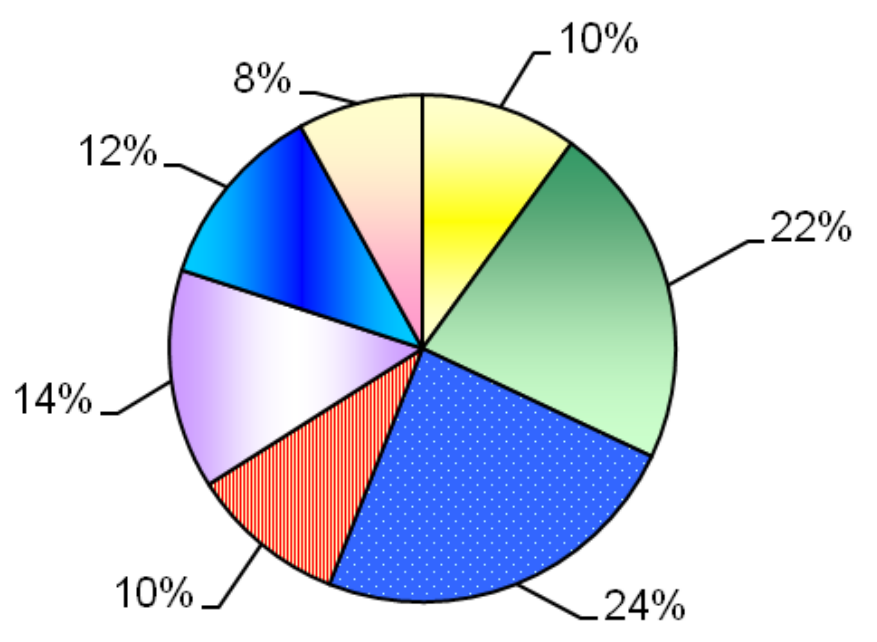

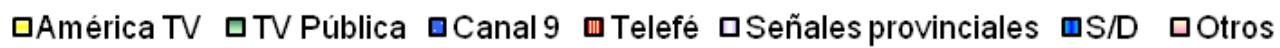

Porcentaje de ficciones emitadas por señal entre 2011-2015 Fuente: elaboración propia

El Trece, perteneciente al Grupo Clarín, a pesar de contar con parte de Pol-Ka -una de las principales productoras de ficción del país-, no participó en las convocatorias. Esto se debe a la relación que el Grupo Clarín mantuvo con el gobierno de Cristina Fernández de Kirchner. TELEFE, en tanto, aunque posee un elevado índice de producción ficcional propia, sí emitió ficciones. América TV y Canal 9, en tanto, no generan contenidos ficcionales en forma habitual por lo que resultaron beneficiados con estos concursos.

La mayoría de las producciones estuvo a cargo de productoras con antecedentes en el campo audiovisual. Algunas, estrictamente vinculadas al sector televisivo (Underground, GP Medios, LC Acción, Promofilm Imagina, Eyeworks 4 Cabezas, Torneos y Competencia, Kapow, Fundación Octubre, E1 Oso Producciones); otras, al sector cinematográfico (Tres mentes S.A., Sudestada Cine S.R.L., Campo Cine, Lagarto Cine, Oruga Films, Haddock Films S.R.L., Azteka Films S.R.L., Historias cinematográficas, Aleph Media, Capital Intelectual); algunas, creadas para la ocasión (Atuel Producciones, Zoelle Producciones, Tostaki SA, Valga SRL, 3C Film SRL); otras, ligadas a actores reconocidos en el campo audiovisual o vinculadas con universidades (Gastón Gularte, Albertina Carri, Universidad Nacional del Litoral, Universidad Nacional del Centro de la Provincia de Buenos Aires). 
Si bien el objetivo de la política de fomento a la producción se basó en favorecer la producción de contenidos federales de calidad para romper con el centralismo, la sistematización evidencia que la mayoría de las productoras estaban ubicadas en la Capital Federal. Esto demuestra las dificultades que se presentan al intentar revertir el centralismo y la concentración de la producción en la zona metropolitana. Una vez relevados los primeros datos ligados a las capacidades instaladas en cada una de las regiones, el desafio consistía en diseñar propuestas que garantizaran modalidades de financiamiento estables y no ligadas a las discrecionalidades y a los presupuestos de los gobiernos de turno.

\section{La deuda de una televisión federal}

Luego de analizar la fase de producción de las ficciones HD, se evalúa. a continuación, la fase de transmisión. Aquí se retoma la arquitectura de la plataforma de la TDT integrada por un total de 16 señales con cobertura nacional. Del análisis se desprende que de las 43 producciones estrenadas entre 2011 y 2015, doce (12) fueron transmitidas por Canal 9, cinco (5) por América TV y cinco (5) por TELEFE. Del resto, una (1) producción fue transmitida por el Canal 10 de Mar del Plata, una (1) por el Canal 12 de Posadas, una (1) por el Canal 9 del Litoral, una (1) por Canal 12 de Trenque Lauquen, dos (2) por Acequia TV Mendoza y una (1) por el Canal 7 de Chubut. Finalmente, por la TV Pública se transmitieron once (11) ficciones, dos (2) se estrenaron en exclusivo por la plataforma CDA, aunque todas estaban disponibles en ese servicio de video on demand de acceso gratuito y financiado con fondos públicos, y una (1) se emitió por Netflix.

De ahí que $51 \%$ de las producciones (22) fueron transmitidas por las señales privadas en abierto que solamente se encuentran disponibles en la plataforma de la TDT en CABA y en AMBA (América TV, Canal 9 y TELEFE), por lo que el resto del país está obligado a suscribirse a un sistema pago. De las ficciones, 25\% (11) se emitió por la TV Pública, 16\% (7) se emitió por canales privados y públicos en abierto de diversas provincias y $7 \%$ se repartió en las plataformas CDA y Netflix. 
Del estudio se desprende una asincronía entre el anuncio de ganadores y la fase de producción y de emisión de los contenidos. Estas dilaciones permiten observar la ausencia de control de los organizadores del concurso hacia las productoras, puesto que una de las condiciones era finalizar y estrenar las ficciones seleccionadas dentro del año de obtenido el premio. Asimismo, se evidencia que las medidas adoptadas no lograron revertir el centralismo en la emisión original de los contenidos, primando una lógica que tendió a favorecer a las principales emisoras televisivas de CABA y de AMBA.

Respecto al rating de las miniseries que ganaron los concursos, las emitidas por las señales privadas han logrado captar mayor audiencia que las emitadas por la señal estatal, una lógica que se hereda del mundo analógico. Aunque ninguna ha superado los dos dígitos, a excepción de Historia de un clan, producida por Underground Producciones y emitida por TELEFÉ. En su primera emisión logró más de 16 puntos de rating y mantuvo los dos dígitos a lo largo de toda su transmisión.

La pantalla de la Televisión Pública (Canal 7) no logró despegar, a pesar de su apuesta a una programación con contenidos y con géneros destinados a públicos y a audiencias diferenciadas (deportes, ficciones, documentales, educativos, infantiles).

La mayoría de las producciones HD fueron emitidas por las señales privadas que la plataforma de la TDA no ofrece hacia todo el país; por el contrario, estas señales solo están presentes en CABA y en AMBA, donde la televisión paga tiene una penetración de $86,7 \%$ y $80 \%$, respectivamente. Por lo cual, el televidente mira estas ficciones a través del sistema pago. En provincias como Santiago del Estero o Misiones estas ficciones no pudieron ser vistas por casi la mitad de los hogares puesto que solo 43,8\% y 50,5\% pagan por mirar televisión, respectivamente. ${ }^{2}$ De esto se infiere que pese a las políticas específicas y a la inyección del financiamiento público, el objetivo de una televisión federal tanto en la producción como en la transmisión transita un camino sigiloso.

La emisión de los contenidos a través de CDA, sin previo paso por un canal de televisión implica dos cuestiones. Por un lado, que se saltea una de las condiciones del concurso según la cual cada producción tenía que presentar un canal asociado para su transmisión. Por otra, la puesta a disposición del contenido en CDA, sin previa transmisión por un canal de televisión, marca 
el desplazamiento hacia un consumo convergente, puesto que el usuario puede seleccionar la pantalla (Smart TV, teléfono móvil, PC, tablet, notebook, netbook) para mirar el contenido siempre que tenga acceso a una conexión de banda ancha. Esto se traduce en una estrategia que se encamina hacia una convergencia en el plano del consumo mediante el aprovechamiento de la plasticidad del producto digital y la multiplicidad de pantallas. Por lo tanto, si se hubiese garantizado una conexión de banda ancha como servicio universal a velocidades óptimas, se podría haber acelerado en la fase de transmisión el saldo de una política audiovisual federal.

Por lo expuesto, se considera que las iniciativas mencionadas fueron prometedoras pero no lograron cumplir con los objetivos de inclusión digital y de federalización. El desafio de la TDA era escindirse de las lógicas productivas ligadas a la dependencia centralista. Así pues, la apuesta de una política tecno-productiva quedó trunca dado que la TDA no logró la autonomía necesaria.

\section{Reflexiones finales}

El trabajo sistematizó una serie de datos vinculados al fomento de la producción de contenidos audiovisuales en el marco de implementación de la TDT y la LSCA. Una de las dimensiones de la política de comunicación se centró en la producción de contenidos. Esto incluía múltiples objetivos, entre ellos, revertir la lógica centralista y promover la inclusión y el federalismo de las propuestas audiovisuales. Además, suponía un estímulo para el desarrollo de fuentes de empleo y el conocimiento de las capacidades tecnológicas instaladas en cada región del país.

Con esta perspectiva, se organizaron una serie de concursos impulsados por el MinPlan, el CIN, la UNSAM y el INCAA. En el estudio se relevaron las series de ficción en alta definición y se identificaron las productoras beneficiadas y las señales para su emisión. A partir de esto, se observa que de las 50 propuestas financiadas $28 \%$ fue para productoras televisivas con antecedentes, radicadas en CABA y AMBA, y 20\% para productoras cinematográficas; $44 \%$ de las realizaciones estuvieron a cargo de productoras audiovisuales, de las cuales algunas se crearon con el fin de participar en las convocatorias, $2 \%$ fueron 
efectuadas por realizadores independientes y $2 \%$ por universidades nacionales. Se infiere, de lo anterior, que la medida impulsó la entrada de nuevos actores provenientes del sector cinematográfico y universitario a la industria televisiva digital. No obstante, no colaboró a instalar los mecanismos necesarios para producir contenidos autosustentables y descentralizados de CABA y AMBA, puesto que $94 \%$ de las propuestas se realizaron en esta zona geográfica.

En el período 2011-2015 se financiaron 50 producciones y se estrenaron 43; 22 se emitieron en las señales privadas de Buenos Aires (América TV, Canal 9, TELEFE) y 11 por la Televisión Pública (Canal 7). Las siete (7) restantes se distribuyeron por Canal 10 de Mar del Plata, Canal 12 de Posadas, Canal 9 del Litoral, Canal 12 de Trenque Lauquen, Acequia TV de Mendoza y Canal 7 de Chubut. En tanto, dos (2) se habian estrenado solamente en la página de Contenidos Digitales Audiovisuales (CDA) y una (1) se emitió por la plataforma de pago Netflix, eludiéndose su estreno por la televisión abierta nacional. De las siete (7) ficciones restantes, sobre seis (6) no se hallaron datos y de una (1) solo se obtuvo el tráiler. Estos datos indican que tampoco se revirtió el centralismo en la emisión de los contenidos, lo que hubiese sido posible puesto que las ficciones fueron financiadas con fondos del erario público.

Este último eje resulta de interés por la reusabilidad de los contenidos. Un producto que se pensó originalmente para la televisión puede circular por múltiples plataformas, incluso algunas ficciones han sido estrenadas exclusivamente en CDA. Esto permite alcanzar economías de escala en la fase de distribución, si se consideran dos factores: las nuevas lógicas de consumo en Internet y que se trata de una industria de prototipo.

Sin embargo, la multiplicidad de pantallas no necesariamente atiende al objetivo de inclusión digital. Primero, porque el acceso a Internet está relacionado con la capacidad de pago del usuario; segundo, porque la mayoría de las series se emitieron por señales privadas que se transmiten por aire y gratuitamente a través de la TDT solo en AMBA y CABA.

En esta dirección, una primera respuesta ante el interrogante sobre si se logró revertir el centralismo en la producción y en la emisión de los contenidos audiovisuales sería que se advierte una predominancia de la Ciudad Autónoma de Buenos Aires. Aunque estas políticas se presentaban como un estímulo para generar capacidades productivas en nuevas áreas geográficas, 
no se logró revertir la lógica centro-periferia. Asimismo, emergieron actores en la industria televisiva que en su mayoria llegaban del cine. Esto puede corresponder a la participación del INCAA y de productoras y directores/as del sector. Como resultado, se establece una convergencia entre la cultura cinematográfica y la televisiva en relación con las formas estéticas y de producción.

Uno de los desafios era la coordinación de las politicas a mediano y a largo plazo, para no quedar sujetos a politicas coyunturales ligadas a las discrecionalidades de los gobiernos de turno. Para ello, resultaba necesario un flujo estable de financiamiento a la producción y el estímulo al autofinanciamiento a través de alianzas con el sector público y de acuerdos de cooperación con el sector privado que fortalecieran el rol de la televisión pública.

En consecuencia, se observa que existió desarticulación, descoordinación y discrecionalidad en el desarrollo de las políticas de comunicación audiovisuales que fueron analizadas, entre la letra de la LSCA y los objetivos de la TDA, lo cual eclipsó su implementación.

\section{Referencias}

AFSCA (2012). Informe Anual sobre Contenidos de la Televisión Abierta [Solicitar a la autora mediante correo electrónico a ornelacarboni@yahoo.com.ar]

Becerra, M. (2016). Restauración: cambios en las políticas de comunicación. Épocas. Revista de Ciencias Sociales y Crítica Cultural. Recuperado de http: / / revistaepocas.com.ar/restauracion-cambios-en-laspoliticas-de-comunicacion/\#_ftn1

Becerra, M. (2011). La incubación de una nueva cultura. Telos, (88) (julio-septiembre) Recuperado de https: / / www.fundaciontelefonica.com.ar/culturadigital/publicaciones-listado/pagina-itempublicaciones /itempubli/256/ 
Becerra, M. y Mastrini, G. (2009). Los dueños de la palabra. Ciudad Autónoma de Buenos Aires, Argentina: Prometeo.

Carrier y asociados (2016). Acceso a Internet y tecnología residencial [Informe]. Ciudad Autónoma de Buenos Aires, Argentina.

Decreto Ley 22.285 de Radiodifusión (1980). Recuperado de http:/ / servicios.infoleg.gob.ar/infolegInternet/anexos / 15000$19999 / 17694 /$ texact.htm

Decreto Nacional 267 (2015). Ente Nacional de Comunicaciones. Boletín Oficial de la República Argentina. Buenos Aires, 04/01/2016. Recuperado de http://servicios.infoleg.gob.ar/infolegInternet/anexos / 255000 $-259999 / 257461 /$ norma.htm

Decreto Nacional 513 (2017). Ley de Ministerios. Boletín Oficial de la República Argentina. Buenos Aires, 17/07/2017.

Recuperado de http:/ / servicios.infoleg.gob.ar/infolegInternet/anexos/275000 $-279999 / 276919 /$ norma.htm

Ley Nacional 26.522 de Servicios de Comunicación Audiovisual (2009). Recuperado de http:/ / servicios.infoleg.gob.ar/infolegInternet/anexos / 155000 $-159999 / 158649 /$ norma.htm

Mosco, V. (2006). La Economía Política de la Comunicación: una actualización diez años después. CIC-Cuadernos de Información y Comunicación, (11), 57-79. Recuperado de https:/ / revistas.ucm.es/index.php/CIYC/article/view/CIYC06 06110057A

Pasquali, A. (1991). El orden reina: escritos sobre comunicaciones. Caracas, Venezuela: Monte Ávila. 
Zallo, R. (1988). Economía de la comunicación y la cultura.

Madrid, España: Akal.

\section{ANEXO 1}

Cuadro con ficciones ganadores, productoras, canales de emisión, fecha del concurso y fecha emisión del contenido (Fuente: elaboración propia).

\begin{tabular}{|c|c|c|c|c|c|}
\hline N. ${ }^{\circ}$ & Título ficción & Productora & Canal de emisión & $\begin{array}{l}\text { Fecha del } \\
\text { concurso }\end{array}$ & $\begin{array}{l}\text { Fecha de } \\
\text { emisión }\end{array}$ \\
\hline 1 & Los sónicos & $\begin{array}{c}\text { GP Media-BBC } \\
\text { Worldwide, INCAA }\end{array}$ & Canal 9 & 2011 & 2011 \\
\hline 2 & Decisiones de vida & LCA Producciones & Canal 9 & 2011 & 2011 \\
\hline 3 & Proyecto Aluvión & MC Producciones & Canal 9 & 2011 & 2011 \\
\hline 4 & $\begin{array}{l}\text { Televisión } \\
\text { por la inclusión }\end{array}$ & $\begin{array}{c}\text { On TV Llorente } \\
\text { y Villarruel Contenidos }\end{array}$ & Canal 9 & 2011 & 2011 \\
\hline 5 & El pacto & Tostaki y Oruga & América & 2011 & 2011 \\
\hline 6 & $\begin{array}{c}\text { Historia } \\
\text { de la primera vez }\end{array}$ & Illusion Studios & América & 2011 & 2011 \\
\hline 7 & $\begin{array}{c}\text { Maltratadas, } \\
\text { su única culpa } \\
\text { es haber nacido mujer }\end{array}$ & Torneos y Competencia & América & 2011 & 2011 \\
\hline 8 & Víndica & Promofilm Imagina & América & 2011 & 2011 \\
\hline 9 & Adictos & LCA Producciones & $\begin{array}{c}\text { Canal } 10 \\
\text { Mar del Plata }\end{array}$ & 2011 & 2011 \\
\hline 10 & El donante & Eyeworks 4 Cabezas & TELEFE & 2011 & 2012 \\
\hline 11 & $\begin{array}{l}\text { Las huellas } \\
\text { del secretario }\end{array}$ & Lagarto Cine & Canal 7 & 2012 & 2013 \\
\hline 12 & Historia clínica & Underground & TELEFE & 2012 & 2013 \\
\hline 13 & La viuda de Rafael & Atuel Producciones & Canal 7 & 2012 & 2012 \\
\hline 14 & Amores de historia & Oruga FIlms S.A. & Canal 9 & 2012 & 2012 \\
\hline 15 & Siete vuelos & Gastón Gularte & Canal 12, Posadas & 2012 & 2012 \\
\hline 16 & $\begin{array}{l}\text { Quién mató } \\
\text { al Bebe Uriarte }\end{array}$ & $\begin{array}{l}\text { Universidad Nacional } \\
\text { del Litoral (UNL), } \\
\text { El Triángulo } \\
\text { Producciones } \\
\text { y Arcadia Consultora } \\
\end{array}$ & Canal 9, Litoral & 2012 & 2013 \\
\hline 17 & La nocturna & Turizia S.A. & cda.gob.ar & 2012 & 2013 \\
\hline 18 & $\begin{array}{l}23 \text { Pares: identidades, } \\
\text { amor y genética }\end{array}$ & $\begin{array}{l}\text { Albertina Carri/Marta } \\
\text { Dillon }\end{array}$ & Canal 9 & 2012 & 2012 \\
\hline 19 & Babylon & GP Media & Canal 9 & 2012 & 2012 \\
\hline 20 & $\begin{array}{l}\text { Embarcados a Europa: } \\
\text { la vuelta a los orígenes }\end{array}$ & Mancha Productora & Canal 7 & 2012 & 2014 \\
\hline 21 & Boyando & Haddock Films S.R.L. & $\begin{array}{c}\text { Canal } 9 \\
\text { de Trenque } \\
\text { Lauquen }\end{array}$ & 2012 & 2013 \\
\hline
\end{tabular}




\begin{tabular}{|c|c|c|c|c|c|}
\hline 22 & Viento Sur & Azteka Films S.R.L. & $\begin{array}{c}\text { Canal } 7 \\
\text { de Chubut }\end{array}$ & 2012 & 2012 \\
\hline 23 & $\begin{array}{c}\text { Televisión por la } \\
\text { justicia/ } \\
\text { Santos y pecadores }\end{array}$ & ON TV & Canal 9 & 2013 & 2013 \\
\hline 24 & $\begin{array}{c}\text { Las } 13 \text { esposas } \\
\text { de Wilson Fernández }\end{array}$ & GP Producciones & Canal 7 & 2013 & 2014 \\
\hline 25 & Fábricas & Unicen & Canal 7 & 2013 & 2015 \\
\hline 26 & $\begin{array}{l}\text { La joven guardia, } \\
\text { una épica del barrio }\end{array}$ & Tostaki S.A. & $\mathrm{S} / \mathrm{D}$ & 2013 & - \\
\hline 27 & Cuestión de identidad & Zarlek Producciones & Canal 7 & 2013 & 2014 \\
\hline 28 & Negro y amarillo & Sinema S.A. & $\mathrm{S} / \mathrm{D}$ & 2013 & - \\
\hline 29 & Vida de película & Tres mentes S.A. & cda.gob.ar & 2013 & 2014 \\
\hline 30 & Celebraciones & Sudestada Cine S.R.L. & $\mathrm{S} / \mathrm{D}$ & 2013 & - \\
\hline 31 & $\begin{array}{c}\text { Coma } \\
\text { (el amor te despierta) }\end{array}$ & Atuel Producciones & Acequia, Mendoza & 2013 & 2014 \\
\hline 32 & La casa & Campo Cine & Canal 7 & 2013 & 2015 \\
\hline 33 & La misión & Professio Divinitus SA & Acequia, Mendoza & 2013 & 2014 \\
\hline 34 & El legado de Garibaldi & Idealismo contenidos & Canal 9 & 2013 & 2014 \\
\hline 35 & El mal menor & Manucorp & Canal 7 & 2013 & 2015 \\
\hline 36 & La celebración & $\begin{array}{l}\text { Underground } \\
\text { Producciones }\end{array}$ & TELEFE & 2013 & 2014 \\
\hline 37 & Cazados & Tronera Producciones & América & 2013 & 2015 \\
\hline 38 & Fronteras & Zoelle Producciones & TELEFE & 2013 & 2015 \\
\hline 39 & Estocolmo & Kapow S.A y Story Lab & Netflix & 2014 & 2016 \\
\hline 40 & Todos comen & Atuel Producciones S.R.L & Trailer & 2014 & - \\
\hline 41 & Conflictos modernos & $\begin{array}{c}\text { Llorente y Villarruel } \\
\text { Contenidos S.A. }\end{array}$ & Canal 9 & 2014 & 2015 \\
\hline 42 & La última hora & $\begin{array}{c}\text { Fundación Octubre } \\
\text { (Gastón Portal) }\end{array}$ & Canal 7 & 2014 & 2016 \\
\hline 43 & Milagros en campaña & $\begin{array}{c}\text { El oso producciones } \\
\text { S.R.L }\end{array}$ & Canal 9 & 2014 & 2015 \\
\hline 44 & Cromo & $\begin{array}{c}\text { Historias } \\
\begin{array}{c}\text { Cinematográficas S.A } \\
\text { (Lucía Puenzo) }\end{array}\end{array}$ & Canal 7 & 2014 & 2015 \\
\hline 45 & $\begin{array}{c}\text { Olimpia, } \\
\text { camino a la gloria }\end{array}$ & $\begin{array}{c}\text { Aleph Media S.A. } \\
\text { (Sokolowicz) }\end{array}$ & $\mathrm{S} / \mathrm{D}$ & 2014 & - \\
\hline 46 & Vida política & Professio Divinitus S.A & $\mathrm{S} / \mathrm{D}$ & 2014 & - \\
\hline 47 & $\begin{array}{l}\text { Una mujer } \\
\text { con sombrero }\end{array}$ & Capital Intelectual S.A. & $\mathrm{S} / \mathrm{D}$ & 2014 & - \\
\hline 48 & Mis noches $\sin \mathrm{ti}$ & Valga SRL & Canal 7 & 2014 & 2017 \\
\hline 49 & Cartoneros & $\begin{array}{l}\text { 3C FILM SRL (Matías } \\
\text { Bertilotti), Marcelo } \\
\text { Altmark y Juan Crespo } \\
\text { (Productores) }\end{array}$ & Canal 9 & 2014 & 2017 \\
\hline 50 & Historia de un clan & $\begin{array}{l}\text { Underground } \\
\text { Producciones }\end{array}$ & TELEFE & 2014 & 2016 \\
\hline
\end{tabular}




\section{Notas}

1 El Decreto 267 relaja la regulación ligada a la concentración de la propiedad en televisión abierta, televisión por cable y radios, extiende el plazo de explotación de todas las licencias audiovisuales y autoriza a los grupos de telecomunicación a brindar televisión por cable en unos años. Asimismo, aumenta de 10 a 15 el número de licencias de radio y de televisión abierta a nivel nacional y de tres a cuatro las licencias en el orden local. La televisión por cable, en tanto, se considera un servicio de TIC y se elimina el limite de $35 \%$ de la cobertura de audiencia potencial para emisores de televisión abierta y radio (Becerra, 2016).

2 Datos obtenidos en www.lamac.org 